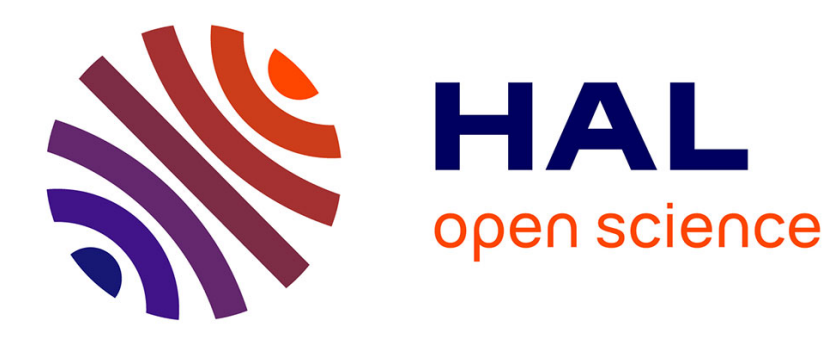

\title{
Pressure effect on electron localization in solid lithium
} Bernard Silvi

\section{To cite this version:}

Bernard Silvi. Pressure effect on electron localization in solid lithium. Structural Chemistry, 2017, pp.1-19. 10.1007/s11224-017-0962-7 . hal-01534558

\section{HAL Id: hal-01534558 \\ https://hal.sorbonne-universite.fr/hal-01534558}

Submitted on 7 Jun 2017

HAL is a multi-disciplinary open access archive for the deposit and dissemination of scientific research documents, whether they are published or not. The documents may come from teaching and research institutions in France or abroad, or from public or private research centers.
L'archive ouverte pluridisciplinaire HAL, est destinée au dépôt et à la diffusion de documents scientifiques de niveau recherche, publiés ou non, émanant des établissements d'enseignement et de recherche français ou étrangers, des laboratoires publics ou privés. 


\title{
Pressure effect on electron localization in solid lithium ${ }^{\star}$
}

\author{
Bernard Silvi
}

Received: date / Accepted: date

\begin{abstract}
External pressure applied to a solid material causes modifications of the bonding which can provide a chemical explanations of phase transitions. The behaviour of the Electron Localization Function $(E L F)$ has been examined for the body centred cubic, face centred cubic and $I \overline{4} 3 d-16$ phase of lithium for a series of cell volumes accounting for external hydrostatic pressures in the 0-60 GPa range. It is shown that the $E L F$ signatures of electron localization increase with the pressure. Moreover, the number of basins per atom is the high pressure phases is less than in the low pressure ones and therefore the basin population are larger in high pressure modifications. These results complement the study of Marques et al. [1] carried out on the $C 2 c b-40$ and Cmca - 24 phases stable above $85 \mathrm{GPa}$
\end{abstract}

Keywords Metallic bond; Electron Localization Function; delocalization; pressure effects

\section{Looking for chemical explanations of crystalline structures}

"Are Crystal Structures Predictable?" is the title of an article published by Angelo Gavezzoti in which he encourages rephrasing the query in terms of specific and more restricted questions in order of increasing complexity[2]. A closely related, but different, question is "How explain crystal structures?". By explain a crystal structure it is meant how and why a crystal structure is observed under given condition. Explanations are not unique and therefore one has to choose a level of explanation. For example we can seek physical explanations based on the lattice energy or chemical explanations relying upon the nature of the elemental atoms comprised in the solid. The behaviour of lithium under pressure provides a very good subject for such an investigation.

* This paper is dedicated to Professor Lou Massa on the occasion of his Festschrift

B. Silvi

Sorbonne Universités, UPMC, Univ Paris 06, UMR 7616, Laboratoire de Chimie Théorique, case courrier 137, 4 place Jussieu, F-75005 Paris, France

Tel.: +33-1-44-274211

Fax: +123-1-44-275526

E-mail: silvi@lct.jussieu.fr 
The structure of solid lithium has been investigated over a wide pressure range. The phase diagram mapped to $130 \mathrm{GPa}[3,1]$ indicates eight observed modifications denoted according to their lattice structure: bcc, $h R 9$, fcc, $h R 1, c I 16(I \overline{4} 3 d), o c 88(C 2 m b), o 40$ $(C 2 c b$ and $\mathrm{Pbca})$ and $C 24(\mathrm{Cmca})$. Moreover, it had been found that the resistivity increases with pressure and that a metal-to-semiconductor transition occurs near 80 GPa[4]. Neaton and Ashcroft have proposed a physical explanation, based on first principle calculations, which invokes the pairing of atoms[5]. The physical origin of this pairing has to be found in the increasing importance of orthogonality and exclusion with rising density which is susceptible to induce a Peierls pairing distortion. The increase of the electron localization at high pressure has been investigated by Rousseau and Marx[6] who carried out Car-Parinello Molecular Dynamics[7] calculations on a large sample of aggregates, bulk phases and SLABs covering a rather large range of nearest neighbour Li-Li distances. The analysis of the Electron Localization Function $(E L F)[8]$ emphasizes the importance of the multicentre interstitial bonding in bulk materials as well as the increase of $E L F$ at the valence attractors when going from a low pressure structure to a higher pressure one. This is confirmed by the analysis made by Marqués et al. [1] with the help of the ELF which evidences the formation of Lewis electron pairs in the interstitial sites of the $C 2 c b-40$ phase.

The present study is inspired by the article of Rousseau and Marx[6]. It aim to show how the topological analysis of $E L F$ provides a consistent background for the emergence of chemical explanations of both structural changes and resistivity evolution in the pressure range $0-60 \mathrm{GPa}$.

\section{Chemical models of the metallic bond meet topological approaches}

The bonding in bulk metals is currently explained by the band structure theory and implies the closure of the energetic gap between the valence and conduction bands $[9$, 10]. This combined energetic-orbital picture is related to the reciprocal space representation of the crystal. In the absence of a direct space representation, the metallic bond is rather difficult to include in any general chemical theory of the bonding. For example, G. N. Lewis has not considered the metallic bond in his classical textbook[11] while Pauling describes it as a partial covalent bond between nearest neighbour atomic centres[12]. This covalent description has been more recently advocated by Anderson et al. [13] and by L. C. Allen and J. Capitani[14] in order to remove the metallic bond from the vocabulary of Chemistry. The simplest model, the jellium model, describes a bulk metal or alloy as a periodic array of positively charged ions embedded in a uniform homogeneous gas which can be understood as an extreme case of delocalization. The interstitial-electron model (IEM) has been developed in the generalized-valence-bond method by Mo and Goddard[15] for lattice dynamics in close-packed structures which considers orbitals centred in the tetrahedral site at equilibrium, but able to shift position adiabatically under lattice vibrations. The partial bond model implies that the valence electron density will be distributed among the first neighbour pairs of atoms whereas, in the interstitial model one has to consider interstitial sites involving at least four atomic centres rather than pairs of atoms. For example, in the bcc structure of lithium the conventional cell contains two atoms and two valence electrons. Each atom has 8 nearest neighbours and therefore the partial bond model yields a picture of 8 eighth electron pairs, in other words $0.25 e^{-}$. There are two type of interstitial sites on the one hand tetrahedral sites centred at position $12 d$ and on the other hand oc- 
tahedral sites centred at positions $6 b$ which are in fact the union of four tetrahedra sharing a common edge on a $C_{4}$ axis. Tetrahedral interstitial bonds are expected to have a population of $1 / 12$ electron pair $\left(0.167 e^{-}\right)$which is summed to $0.667 e^{-}$for the octahedral ones. Moreover, each face is shared by two tetrahedra. In order to characterize the bonding it is necessary to consider the pieces of information provided by the electron density of probability functions.

The Quantum Theory of Atoms in Molecules (QTAIM) )[16] is based on the topological analysis of the gradient vector field of the electron density by the dynamical system theory toolbox. It enables both a qualitative and quantitative characterization of the bonding in molecules and solids. It provides a partition of the geometric space in terms of basins of attractors (local maxima of the function) bounded by zero flux surfaces of the gradient of the one-electron density. Attractors are generally located at the nuclear positions, however non-nuclear attractors (NNAs) are found for internuclear separations which are generally far from equilibrium distances[17] except for metal element diatomic molecules[18,19] and bulk metals[20,22,23]. The QTAIM basins are called atomic basins in the absence of NNAs. They are open quantum systems which satisfy a local virial theorem[16,24] enabling a consistent energy partitioning in terms of atomic contributions by the Interacting Quantum Atoms (IQA) approach[29]. The description of the matter given by the QTAIM approach in terms of space-filling non overlapping atoms departs from the representation adopted in chemistry in which bonded atom may share groups of valence electrons. In fact, the QTAIM considers the concept of chemical bond as unphysical and unnecessary. Instead, the structure is accounted for by the molecular graph which consists in the set of bond paths. A bond path connects two nuclei by the trajectories of the gradient field linking a saddle point of index 1 (maximum in two directions, minimum in one) called bond critical point (bcp) to the attractors located at the nuclear positions[33]. The QTAIM characterizes further the interactions by considering the values of quantities evaluated at the bcp, for example: the electron density, its laplacian , the energy density. Several bonding classifications have been proposed accordingly[34] as a metallicity index[42]. These bond indexes are well suited for the description of two-centre bonds but they are not able to characterize multicentre bonds.

In chemistry we are used to thinking the matter not only in terms of atoms but also in terms of bond and lone pairs, concepts which do not emerge from quantum mechanics. They belong to a successful explanatory representation of the matter designed by the chemistry community in which groups of electrons account for the structural and chemical properties. Electron count is therefore essential in chemical explanation of the structure and reactivity. Nevertheless, it is possible to assume that electrons are localized within space-filling non-overlapping domains. The electron count is achieved with the help of a population operator $\hat{N}(\Omega)[43]$ and the variance of the expectation value, the domain population $\bar{N}(\Omega)$, is provided by another operator. If the assumption is verified it is possible to determine domain boundaries which minimize the variance of $\operatorname{bar} N(\Omega)[44,45]$. The Electron Localization Function $(E L F)$ has been designed by Becke and Edgecombe to identify "localized electronic groups in atomic and molecular systems"[8]. ELF fairly reproduces the shell structure of atoms even for heavy atoms for which other method fails. Many interpretations of $E L F$ have been given so far in order to get expressions beyond the HF approximation or to provide relationships with other theoretical tools. Savin et al. have demonstrated that the $E L F$ formula can be extended to DFT and Kohn-Sham orbitals. In this case the ELF kernel has the physical meaning of the ratio of the local excess kinetic energy density for the actual system 
and for the same density jellium. Orbital-based interpretations of $E L F$ have been proposed by Burdett[46] and by Nalewajski et al. [47], who considered the non-additive interorbital Fisher information. Another route pioneered by Dobson[48] explicitly considers the pair functions. It has been independently developed by Kohout et al. with the Electron Localization Indicator (ELI) [49,50] and by me [51], with the spin pair composition $c_{\pi}(\mathbf{r})$ enabling to generalize $E L F$ to correlated wave functions[52,53]. Applying the gradient dynamical system partitioning technique to the $E L F$ yields basins of attractors which correspond to cores, lone pairs and bond regions[54,55]. As noted by Gillespie and Robinson: "This function $(E L F)$ exhibits maxima at the most probable positions of localized electron pairs and each maximum is surrounded by a basin in which there is an increased probability of finding an electron pair. These basins correspond to the qualitative electron pair domains of the VSEPR model and have the same geometry as the VSEPR domains."[56] The basins of the gradient field of $E L F$ faithfully match the density partition of the Lewis's model. On the one hand are the core basins which gather the electron density of the inner atomic shells and on the other hand the valence basins accounting for the bond, lone pair and single electron domain. The core basins, labellled as $\mathrm{C}(\mathrm{A})$ where $\mathrm{A}$ is the atomic symbol of the element, surround nuclei with atomic charge $Z>2$. The number of core basins varies with the number of core shell of the element and also with the local symmetry in the molecule. For isolated atoms the spherical symmetry implies that the attractors corresponding to the electron pairs of the $\mathrm{L}, \mathrm{M}, \ldots$ inner shells are degenerated on a sphere. There is therefore one basin for each shell. In molecules, the symmetry being lower than in isolated atoms, the number of core basins of atoms heavier than neon is larger than 1 and it is convenient to gather them in a single superbasin. In the spirit of Lewis's model, the valence basins are characterized by the atomic valence shells to which they participate, or in other words by the core basins with which they share a boundary. The number of such atomic valence shells is called the synaptic order. Thus, there are monosynaptic, disynaptic, trisynaptic basins and so on. Monosynaptic basins, labelled $\mathrm{V}(\mathrm{A})$, correspond to the lone pairs of the Lewis model, and polysynaptic basins to the shared pairs of the Lewis model. In particular, disynaptic basins, labelled V(A, B) correspond to two-centre bonds, trisynaptic basins, labelled V(A, B, C) to three-centre bonds and so on. The concept of localization domain has been introduced[57] in order to discuss $E L F$ isosurface graphical representations and also to i define a hierarchy of the localization basins which can be related to chemical properties. A localization domain is defined as a volume limited by one or more closed isosurfaces $E L F(\mathbf{r})=f$. It surrounds at least one attractor, in this case it is called irreducible, whereas if it contains more than one attractor, it is said reducible. The increase of the bounding isosurface value splits the reducible domain into domains containing fewer attractors than the parent domain. The reduction of localization occurs at turning points which are index 1 critical points, located on the separatrix of the two basins involved in the parent domain. These critical points are called basin interconnection points often abbreviated by bips[58]. Ordering these turning points (localization nodes) by increasing $\eta(\mathbf{r})$ enables to build tree-diagrams reflecting the hierarchy of the basins[59]. The ELF population analysis is carried out by integrating the electron density over the basin volumes. It is completed by a variance analysis which help to understand the delocalization. In hydrogen bonded complexes, the $E L F$ value at the bip between the $\mathrm{V}(\mathrm{A}, \mathrm{H})$ and $\mathrm{V}(\mathrm{B})$ basins of proton donor and proton acceptor moieties is fairly correlated with the variance of the populations of these moieties[60]. 
The jellium model implies that the density outside of the core region is constant and therefore its gradient at any point should be zero as well as all successive derivatives and therefore all the points are said non-hyperbolic because the eigenvalues of the second derivative (Hessian) matrix of the density are zero. The gradient dynamical system of the density is structurally unstable[61] which is not the case in areal crystal. The removal of the instability yields a finite number of hyperbolic critical points in each cell, but not necessarily of NNAs. However, the jellium model tells us that the values of the density function at the off-core critical points are confined in a very narrow range independently from their indexes. The averaged value of the off-core density can be estimated as the ratio of the number of valence electrons by the atomic volume. In the case of the analysis of the electron localization function, the jellium model also gives rise to a continuum of non-hyperbolic critical points discretized by the improvement of the model. However, there are necessarily valence basins and therefore valence attractors. The value of $E L F$ at the saddle points connecting the valence basins, $E L F_{v v}$, defines the reducible localization domain which extends over the whole crystal. It is expected to be very little less than that valence attractor $E L F\left(\mathbf{r}_{a}\right)$ and the interval $\left[E L F_{v v}, E L F\left(\mathbf{r}_{a}\right)\right]$ defines the "localization window"[22]. The location of the $E L F$ valence maxima provide strong arguments to discriminate the partial covalent and interstitial bond pictures. The valence attractors are expected to be close the first neighbour line midpoint when the partial covalent model is dominating and otherwise close to interstitial positions.

\section{A numerical study of the bcc, fcc and $I \overline{4} 3 d$ phases of $L i$ in the 0-50 GPa} pressure range

The calculations have been performed at the DFT level with the periodic program CRYSTAL98[62]. The basis functions are derived from the TZV set of Schäfer et al. [63] in which the outermost diffuse $s$ function is removed yielding a $\{6211\}$ contraction scheme in which the exponent of the external orbital is set to $\alpha_{s_{10}}=0.1$. Two sets $p$ polarization functions $\left(\alpha_{p_{1}}=0.514\right.$ and $\alpha_{p_{2}}=0.10$ are added and the final contraction scheme is $\{6211 / 11\}^{1}$ These exponents have been chosen in order to avoid linear dependency of the basis set for small atomic volumes. The DFT calculation is done with the exchange-correlation functional of Perdew et al. [64]. For all structures and all atomic volumes, the system is calculated to be conductor. The QTAIM and ELF analyses have been made with TOPOND98[65] and a periodic version of TopMoD[66] and the graphical representations with the Amira software[67]. The basis set used for the calculation provide very satisfactory results for the calculated values of the cohesive energy, zero-pressure atomic volume, bulk modulus and bulk modulus pressure derivative of the bcc phase. The cohesive energy (without zero point motion correction) is given by the difference of the lattice and free atom energy. An upper bound is given by considering the periodic calculation basis set for evaluating the free atom energy whereas the original atomic TZV basis provide a lower bound. The experimental value, $1.63 \mathrm{eV}[68]$, is very close to $1.68 \mathrm{eV}$, the average of the calculated bounds 1.28 and $2.04 \mathrm{eV}$, respectively. The equation of state parameters are not too much sensitive to the type of equation used for the fit. The zero-pressure atomic volume ranges from

\footnotetext{
1 The notation $\left\{s_{1}, s_{2}, s_{3}, \ldots / p_{1}, p_{2}, \ldots / d_{1}, \ldots\right\}$ specifies the number of primitives of each type in the contraction.
} 
$20.809 \AA^{3}$ (Vinet) to $21.105 \AA^{3}$ to be compared to the experimental value 21.6225 $\AA^{3}[69]$. The bulk modulus and its pressure derivative calculated by the Vinet fit are 13.78 GPa and 3.39 whereas the Murnaghan equation fit yields $10.389 \mathrm{GPa}$ and 3.49 both in reasonable agreement with the experimental values, $11.32 \mathrm{GPa}$ and 3.62[69]. The experimental equation of state of Hanfland et al. is used all along this paper to determine de pressure.

\subsection{Basis functions and structural stability}

The bcc phase of lithium has been the topic of several papers reporting both QTAIM and $E L F$ studies in which the location of the NNAs and $E L F$ valence attractors have been shown to strongly depend upon the level of calculation. The Hartree-Fock calculation of Mei et al. [20] carried out with a [(2s)1sp] contraction reports a network of NNAs located at the bond midpoints in position $(8 c)$ whereas they are in tetrahedral interstitial sites $(12 d)$ for a density evaluated with a split-valence basis set[22]. The $E L F$ valence attractors are found in position (6b) by periodic Hartree-Fock[22] and TB-LMTO-ASA[70] or in (12d) by FPLO calculations[70]. The basis set used in his work puts the NNAs and the $E L F$ valence attractors both in position $(12 d)$. Table 1 displays the locations of the NNAs and $E L F$ valence attractors for a representative selection of values of the external basis function exponents. It shows that rather small variation of the exponents are responsible for rather dramatic qualitative as well as quantitative changes in the topology of the valence density. It worth noting that the value of $E L F$ noticeably depends upon the basis set in metallic crystals while its value at valence attractors is almost basis set free in molecules. The use of the same basis set for all modifications and cell parameters is therefore necessary to ensure the reliability of the trends of the calculated electron localization indicators.

Table 1: NNAs and $E L F$ valence attractor positions, density Laplacian at NNA and $E L F$ at attractor

\begin{tabular}{cccccc}
\hline$\alpha_{s}$ & $\alpha_{p}$ & \multicolumn{2}{c}{ NNAs } & \multicolumn{2}{c}{$E L F$ attractors } \\
& & position & $\nabla^{2} \rho\left(\mathbf{r}_{c}\right) \times 10^{-3}$ & position & $E L F$ \\
\hline 0.10 & 0.08 & $(12 d)$ & -1.440 & $(12 d)$ & 0.635 \\
0.10 & 0.10 & $(12 d)$ & -0.907 & $(12 d)$ & 0.616 \\
0.10 & 0.12 & $(24 h)$ & -0.587 & $(12 d)$ & 0.593 \\
0.10 & 0.14 & $(8 c)$ & -0.263 & $(48 k)$ & 0.571 \\
0.10 & 0.16 & $(8 c)$ & -0.320 & $(8 c)$ & 0.606 \\
0.12 & 0.12 & $(8 c)$ & -0.217 & $(12 d)$ & 0.530 \\
0.14 & 0.14 & $(8 c)$ & -3.135 & $(8 c)$ & 0.580 \\
\hline
\end{tabular}

\subsection{The NNAs}

NNAs are found for all structures over the whole range of pressure investigated. The Laplacian of the electron density provides a measure of the curvature of this function at its maxima: larger is its absolute value more peaked is the maximum. In the bcc phase, they are located in tetrahedral interstitial positions $(12 d)$, in the fcc one at 
both octahedral $(4 b)$ and tetrhadral $(8 c)$ and at both $(12 a)$ (octahedral sites) and (12b) (tetrahedral) sites in the $I \overline{4} 3 d$ modification. Fig. 1 displays the evolution of the Laplacian of $\rho\left(\mathbf{r}_{c}\right)$ between 0 and $60 \mathrm{GPa}$.

For all types of NNAs, the Laplacian of $\rho\left(\mathbf{r}_{c}\right)$ varies linearly with the pressure with a negative slope indicating that the NNAs are more marked at high pressures. The largest slope absolute value corresponds to the NNAs located at positions (12a) of the $I \overline{4} 3 d$ phase. Note that in the fcc phase there is no NNA in position $(4 b)$ below 9.0 $\mathrm{GPa}$. Below this pressure, this pressure the critical points in (4b) become local minima. Fig. 1 suggests that presence of NNAs is ruled by their distance to at least two lithium centres: above a critical distance, for example $2.25 \AA$ for the $(4 b)$ positions of fcc. The behaviour of the interstitial NNAs of the investigated phases of lithium is consistent with the predictions of Martin Pendás et al. [17].

\subsection{ELF valence maxima, localization windows and core compressibility}

For all pressure and all phases, the valence maxima of $E L F$ are found is the same interstitial positions that the NNAs. The values of $E L F$ at these attractors are always significantly larger than 0.5 , the value expected for the homogeneous electron gas. The excess kinetic energy of $E L F[71-73]$ implies that in regions of $E L F>0.5$ the Pauli repulsion is lower than in a homogeneous electron gas of the same density. This point has been discussed by Kohout et al. who concluded that $E L F$ does not mirror the Pauli repulsion[58].

Fig. 2 display the $E L F$ isosurfaces below and above the $E L F$ value of the bifurcation which splits the parent reducible valence domain extending over the whole crystal. The irreducible valence domains have almost the shape of the dual polyhedra of the interstitial voids with their faces in front of the lithium centres defining the interstice: for example the octahedral sites of the fcc phase give rise to cubic-like irreducible domains. The critical point connecting two irreducible valence domains one another is located at the common vertex of the two dual polyhedra. In the bcc structure, these point connect tetrahedra, in the fcc one cube to tetrahedra and in the $I \overline{4} 3 d$ pentagonal prisms one another as the critical points linking tetrahedral domains to pentagonal prisms correspond to a lower $E L F$ value. The width of the localization window is defined as the difference of the highest $E L F$ value of the valence attractor and valence-valence bifurcation $E L F$ value. Fig. 3 displays the attractor and valence-valence bifurcation $E L F$ values as functions of the pressure. In the bcc structure where all attractors are located at the same special position, the $E L F$ value at the attractors as well as that of the valence-valence bifurcation increase with pressure. In fcc and $I \overline{4} 3 d$ phases the two sets of attractors behave quite differently: The $E L F$ at the attractors located in the octahedral (fcc and $I \overline{4} 3 d$ ) sites always increases with pressure. For those in the tetrahedral interstices of the fcc structure it increases below 20GPa and remains almost constant between 20 and $40 \mathrm{GPa}$, in the $I \overline{4} 3 d$ structure, the $E L F$ at these attractors decreases with pressure. The valence-valence bifurcations occur at $E L F$ values which behave like those of the lowest $E L F$ attractors. In all cases, the gap between the upper full line and the dashed line which represents the localization window increases with pressure indicating that the electron density acquires locally a pronounced pair character. The valence-valence bifurcation $E L F$ value correlates with the variance of the population in the case of hydrogen bonded complexes[60], higher is this value larger is the variance of the sum of the basin populations of one of the moieties. Assuming 


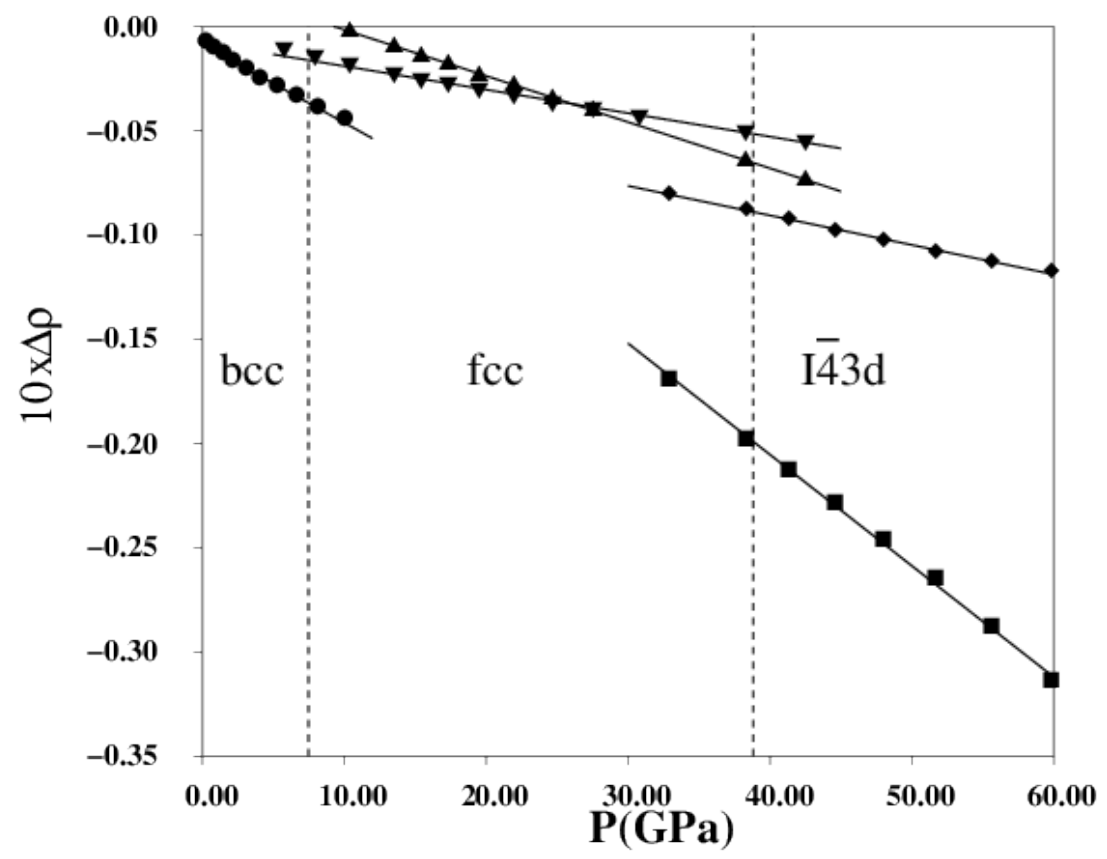

Fig. 1: $\Delta \rho\left(\mathbf{r}_{c}\right)\left(e^{-}\right.$bohr $\left.^{-5}\right)$ v.s. $\mathrm{P}(\mathrm{GPa})$. •: bcc, $\mathbf{\Delta}:$ fcc $(4 b), \mathbf{\nabla}:$ fcc $(12 d), \mathbf{\square}: I \overline{4} 3 d$ $(12 a), \diamond: I \overline{4} 3 d(12 b)$ 


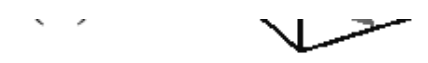

(c)

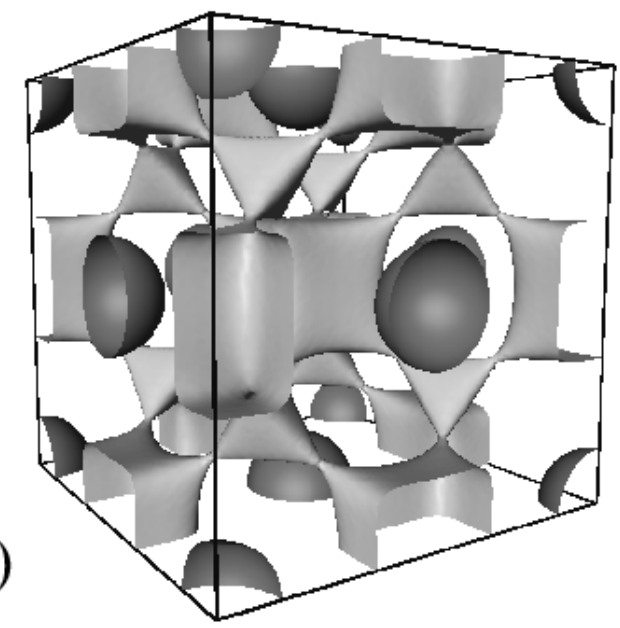

(e)

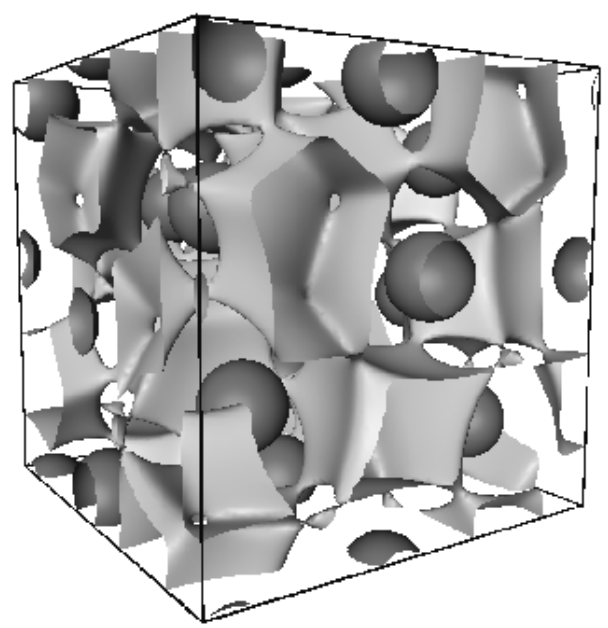

(d)

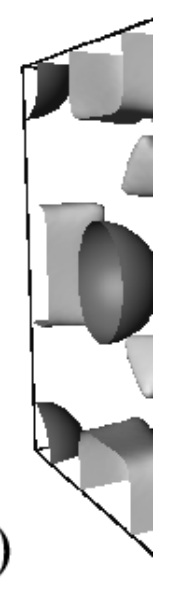

(f)

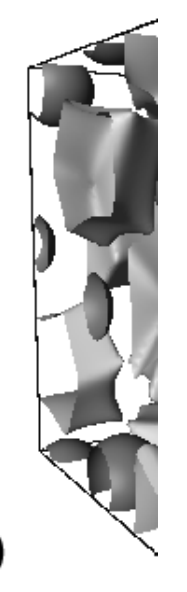

Fig. 2: $E L F$ isosurfaces of the bcc, fcc and $I \overline{4} 3 d$ phases of lithium crystal. (a) bcc $E L F=0.584$, (b) bcc $E L F=0.585$, (c) fcc $E L F=0.615$ (d) fcc $E L F=0.620$, (e) $I \overline{4} 3 d E L F=0.530$, (f) $I \overline{4} 3 d E L F=0.532$. 


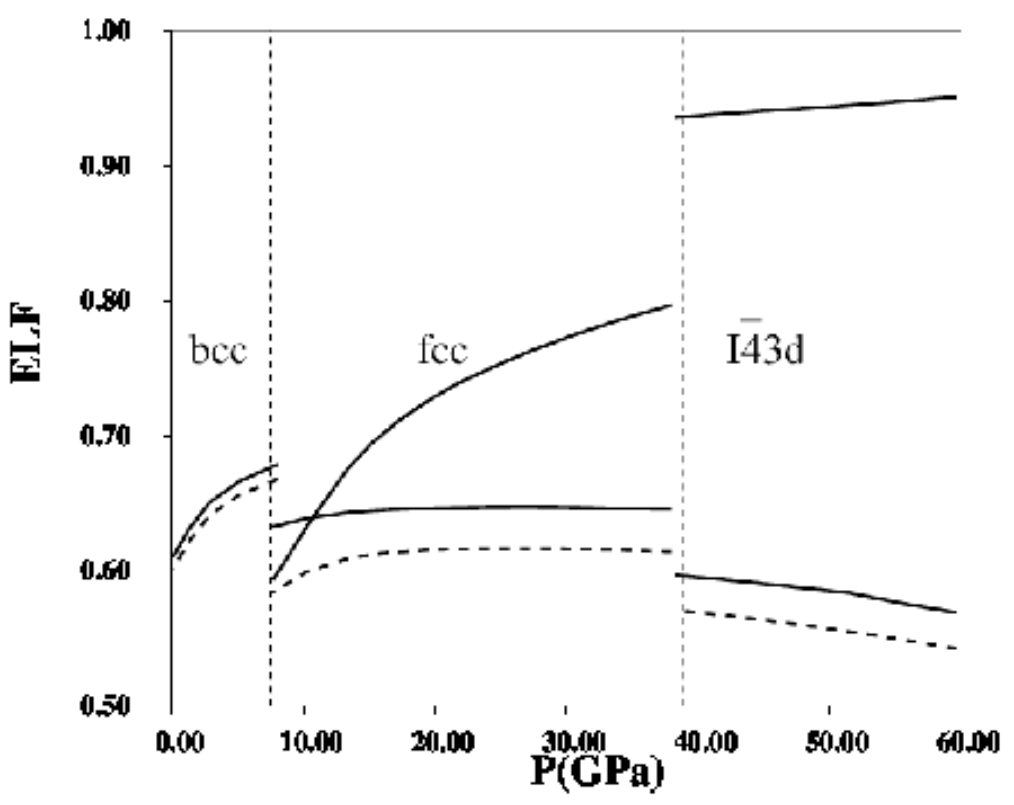

Fig. 3: Attractor (full lines) and valence-valence bifurcation (dashed lines) ELF values vs. pressure. 
that this correlation holds, the delocalization decreases with pressure in the only case of the $I \overline{4} 3 d$ structures whereas it increases for the bcc and fcc phases which appears in contradiction with the strengthening of the pair character. However, the assumption is not true for basin populations less than $1 e^{-}$, for example in the $\mathrm{Li}_{4}$ prototype molecule which is calculated to have a planar rhomboidal $D_{2 h}$ structure with edges of $3.05 \AA$, whereas the distance between the nearest opposite vertices is shorter, i.e. 2.62 $\AA[74]$. Four valence attractors are located, outside of the rhombus, on the bisection of each edge. There are in principle four disynaptic basins $\mathrm{V}(\mathrm{Li}, \mathrm{Li})$, each with a population $\bar{N}[\mathrm{~V}(\mathrm{Li}, \mathrm{Li})]=0.98 e^{-}$. The value of the covariance matrix elements with the two neighbouring $\mathrm{V}(\mathrm{Li}, \mathrm{Li})$ basins are -0.39 when the two basins belong to the valence shell of a $\mathrm{Li}$ at an acute angle vertex and -0.5 when they are in an obtuse angle vertex. The $E L F$ value at the saddle point between two such disynaptic basins is 0.994 to be compared to 0.996 , the value at the attractors.

The ratio of the core and atomic volume increases with pressure independently of the crystalline structure whereas the core population remains almost constant, volumes represent $10 \%$ of the cell volume at $0 \mathrm{GPa}$ and $18.5 \%$ at $60 \mathrm{GPa}$ whereas the core populations remains constant, $2.023 e^{-}$over the investigated pressure range. The compressibility $\beta$ can be expressed in terms of the sum of basin contributions $[75,76]$ :

$$
\beta=-\frac{1}{V} \frac{\partial V}{\partial P}=\sum_{i} f_{i} \beta_{i}
$$

where $f_{i}=V_{i} / V$ is the fractional occupation volume of the $i$-th basin and $\beta_{i}=$ $-\left(1 / V_{i}\right)\left(\partial V_{i} / \partial P\right)$ is the basin compressibility. The ratio of the core and atomic compressibilities which is 0.35 at $0 \mathrm{GPa}$ reaches 0.47 at $60 \mathrm{GPa}$.

\section{Implications of interstitial bonding}

The ELF propose an interstitial bonding picture in which the localization increases with the pressure. In the low pressure regime, the volume of the conventional bcc can be decomposed into three octahedra centred at the $6 b$ positions, these octahedra can be further split into four tetrahedra around the $24 h$ positions. The two valence electrons are distributed among twelve tetrahedra each with a population of $1 / 6 e^{-}$, this implies a rather large variance of the population. A lower bond can be estimated assuming that at most one electron can be counted in a tetrahedron with a probability of $1 / 6$ which yield the variance $\sigma^{2}=1 / 6-1 / 36=5 / 36$. The octahedral interstice can be considered instead of the tetrahedral ones. In this case the population is $2 / 3 e^{-}$and the lower bound of the variance is 0.222 . In the fcc cell four valence electrons are distributed among four octahedra and eight tetrahedra which do not overlap. Each tetrahedron is surrounded by four octahedrons by common faces, The octahedron develop a network connected by tetrahedra. Assuming the valence density constant, the populations of the basins of octahedral and tetrahedral interstices are respectively estimated to be $2 / 3$ and $1 / 3 e^{-}$from the volumes of the interstices. In the $I \overline{4} 3 d$ lattice, the nuclei are in (16c) whereas the attractors of the tetrahedral and octahedral basins are in (12a) and (12b) respectively. The sixteen electrons are distributed among the twelve tetrahedral and twelve octahedral interstices; considering their respective sizes their populations are expected to be respectively $1 / 3^{-}$and $1 e^{-}$. The study carried out by Marqués et al. [1] on the phases over $75 \mathrm{GPa}$ are in line with the results obtained for the lower pressure phases. 
Compliance with Ethical Standards

This theoretical work meets the requirements of the ethical stantards.

\section{References}

1. Marqués M, McMahon MI, Gregoryanz E, Hanfland M, Guillaume CL, Pickard CJ, Ackland GJ, Nelmes RJ (2011) Crystal Structures of Dense Lithium: A Metal-SemiconductorMetal Transition Phys Rev Lett 106:095502

2. Gavezzotti A (1994) Are Crystal Structures Predictable? Acc Chem Res 27:309-314

3. Guillaume CL, Gregoryanz E, Degtyareva O, McMahon MI, Hanfland M, Evans S, Guthrie M, Sinogeikin SV, Mao HK (2011) Cold melting and solid structures of dense lithium Nat Phys 7:211-214

4. Matsuoka T, Shimizu K (2009) Direct observation of a pressure-induced metal-tosemiconductor transition in lithium Nature 458:186-189

5. Neaton JB, Ashcroft NW (1999) Pairing in dense lithium Nature 400:141-144

6. Rousseau R, Marx D (2000) Exploring the Electronic Structure of Elemental Lithium: From Small Molecules to Nanoclusters, Bulk Metal, and Surfaces Chem Eur J 6:29822993

7. Car R, Parrinello M (1985) Unified Approach for Molecular Dynamics and DensityFunctional Theory Chem Phys Lett 2471-2474

8. Becke AD, Edgecombe KE (1990) A simple mesure of electron localization in atomic and molecular systems J Chem Phys 92:5397-5403

9. Kittel C (1963) Quantum Theory of Solids John Wiley and sons, New York

10. Ashcroft NW, Mermin ND (1976) Solid State Physics Saunders College Publishing

11. Lewis GN (1966) Valence and the Structure of Atoms and Molecules Dover, New York

12. Pauling L (1948) The Nature of the Chemical Bond Cornell University Press, Ithaca

13. Anderson WP, Burdett JK, Czech PT (1994) What Is the Metallic Bond? J Am Chem Soc 116:8808-8809

14. Allen LC, Capitani JF (1994) What is the Metallic Bond? J Am Chem Soc 116:8810-8810

15. Li M, Goddard III WA (1989) Interstitial-electron model for lattice in fcc metals Phys Rev B 40:12155-12163

16. Bader RFW (1990) Atoms in Molecules: A Quantum Theory Oxford Univ. Press, Oxford

17. Martín Pendás A, Blanco MA, Costales A, Mori Sánchez P, Luaña V (1999) Non-nuclear Maxima of the Electron Density Phys Rev Lett 83:1930-1933

18. Cao WL, Gatti C, MacDougall PJ, Bader RFW (1987) On the presence of nonnuclear attractors in the charge distribution of $\mathrm{Li}$ and $\mathrm{Na}$ cluster Chem Phys Lett 141:380-385

19. Cioslowski J (1990) Nonnuclear Attractors in the $\mathrm{Li}_{2}$ Molecule J Phys Chem 94:5496-5498

20. Mei C, Edgecombe KE, Smith Jr VH, Heilingbrunner A (1993) Topological analysis of the charge density of solids: bcc sodium and lithium Int J Quant Chem 48:287-293

21. Iversen BB, Larsen FK, Souhassou M, Takata M (1995) Experimental evidence for the existence of non-nuclear maxima in the electron-density distribution of metallic beryllium. A comparative study of the maximum entropy method and the multipole refinement method Acta Cryst B 51:580-591

22. Silvi B, Gatti C (2000) Direct space representation of the metallic bond. J Phys Chem A 104:947-953

23. Baranov AI, Kohout M (2008) Electron localizability for hexagonal element structures J Comput Chem 29:2161-2171

24. Bader RFW (1994) Principle of stationary action and the definition of a proper open system Phys Rev B 49:13348-13356

25. Bader RFW (2001) The zero-flux surface and the topological and quantum definition of an atom in a molecule Theor Chem Acc 105:276-283

26. Bader RFW (2005) The Quantum Mechanical Basis of Conceptual Chemistry Monatshefte für Chemie 136:819-854

27. Bader RFW (2007) Everyman's Derivation of the Theory of Atoms in Molecules J Phys Chem A 111:7966-7972

28. Bader RFW (2007) The Lagrangian Approach to Chemistry in Matta CF, Boyd RJ (eds.) The Quantum Theory of Atoms in Molecules: From Solid State to DNA and Drug Design 37-59 John Wiley \& Sons Inc, New York 
29. Martín Pendás A, Blanco MA, Francisco E (2004) Two-electron integrations in the quantum theory of atoms in molecules J Chem Phys 120:4581-4592

30. Martín Pendás A, Francisco E, Blanco MA (2005) Two-electron integrations in the Quantum Theory of Atoms in Molecules with correlated wave functions J Comput Chem 26

31. Blanco MA, Martín Pendás A, Francisco E (2005) Interacting Quantum Atoms: A Correlated Energy Decomposition Scheme Based on the Quantum Theory of Atoms in Molecules J Chem Theory Comput 1:1096-1109

32. Francisco E, Martín Pendás A, Blanco MA (2006) A Molecular Energy Decomposition Scheme for Atoms in Molecules J Chem Theory Comput 2:90-102

33. Bader RFW (1998) A Bond Path: A Universal Indicator of Bonded Interactions. J Phys Chem A 102:7314

34. Bader RFW, Essén H (1984) The characterization of atomic interactions J Chem Phys 80:1943-1960

35. Cremer D, Kraka E (1984) Chemical Bonds without Bonding Electron Density - Does the Difference Electron-Density Analysis Suffice for a Description of the Chemical Bond? Angew Chem Int Ed Engl 23:627-628

36. Cremer D, Kraka E (1983) A description of the Chemical Bond in Terms of Local Properties of the Electron Density and Energy Croat Chem Acta 57:1259-1281

37. Kraka E, Cremer D (1992) Description of chemical reactions in terms of the properties of the electron density J Mol Struct (Theochem) 255:189-206

38. Macchi P, Proserpio DM, Sironi A (1998) Experimental Electron Density in a Transition Metal Dimer: Metal-Metal and Metal-Ligand Bonds J Am Chem Soc 120:13429-13435

39. Macchi P, Sironi A (2003) Chemical bonding in transition metal carbonyl clusters: complementary analysis of theoretical and experimental electron densities. Coord Chem Rev 238-239:383-412

40. Bianchi R, Gervasio G, Marabello D (2000) Experimental Electron Density Analysis of $\mathrm{Mn}(\mathrm{CO})_{10}$ : Metal-Metal and Metal-Ligand Bond Characterization Inorg Chem 39:23602366

41. Mori-Sánchez P, Martín Pendás A, Luaña V (2002) A Classification of Covalent, Ionic, and Metallic Solids Based on the Electron Density J Am Chem Soc 124:14721-14723

42. Jenkins S, Ayers P, Kirk S, Mori-Sánchez P, Martín Pendás A (2009) Bond metallicity of materials from real space charge density distributions Chem Phys Lett 471:174-177

43. Diner S, Claverie P (1976) Statistical and Stochastic Aspects of the Delocalization Problem in Quantum Mechanics in Chalvet O, Daudel R, Diner S, Malrieu JP (eds.) Localization and Delocalization in Quantum Chemistry vol. II 395-448 Reidel, Dordrecht

44. Silvi B, Fourré I, Alikhani E (2005) The Topological Analysis of the Electron Localization Function : a Key for a Position Space Representation of Chemical Bonds Monatshefte für Chemie 136:855-879

45. Ayers PW (2005) Electron localization functions and local measures of the covariance J Chem Sci 117:441-454

46. Burdett JK, McCormick TA (1998) Electron Localization in Molecules and Solids: The Meaning of ELF J Phys Chem A 102:6366-6372

47. Nalewajski RF, Koster AM, Escalante S (2005) Electron Localization Function as Information Measure J Phys Chem A 109:10038-10043

48. Dobson JF (1991) Interpretation of the Fermi hole curvature J Chem Phys 94:4328-4333

49. Kohout M, Pernal K, Wagner FR, Grin Y (2004) Electron localizability indicator for correlared wavefunctions. I. Parallel spin pairs Theor Chem Acc 112:453-459

50. Wagner FR, Bezugly V, Kohout M, Grin Y (2007) Charge decomposition analysis of the electron localizability indicator: A bridge between the orbital and direct space representation of the chemical bond Chem Eur J 13:5724-5741

51. Silvi B (2003) The Spin Pair Compositions as Local Indicators of the Nature of the Bonding J Phys Chem A 107:3081-3085

52. Matito E, Silvi B, Duran M, Solà M (2006) Electron localization function at the correlated level J Chem Phys 125:024301

53. Feixas F, Matito E, Duran M, Solá M, Silvi B (2010) Electron Localization Function at the Correlated Level: A Natural Orbital Formulation J Chem Theory Comput 6:2736-2742

54. Silvi B, Savin A (1994) Classification of chemical bonds based on topological analysis of electron localization function Nature 371:683-686

55. Häussermann U, Wengert S, Nesper R (1994) Unequivocal Partitioning of Crystal Structures. Exemplified by Intermetallic Phases Containing Aluminium. Angew Chem Int Ed Engl 33:2073-2076 
56. Gillespie RJ, Robinson EA (2007) Models of Molecular Geometry J Comput Chem 28:8797

57. Savin A, Silvi B, Colonna F (1996) Topological analysis of the electron localization function applied to delocalized bonds Can J Chem 74:1088-1096

58. Kohout M, Wagner FR, Grin Y (2002) Electron localization function for transition-metal compounds Theor Chem Acc 108:150-156

59. Calatayud M, Andrés J, Beltrán A, Silvi B (2001) The hierarchy of localization basins: a tool for the understanding of chemical bonding exemplified by the analysis of the $\mathrm{VO}_{\mathrm{x}}$ and $\mathrm{VO}_{\mathrm{x}}^{+}(x=1-4)$ systems. Theoret Chem Acc 105:299-308

60. Silvi B, Ratajczak H (2016) Hydrogen bonding and delocalization in the ELF analysis approach. Phys Chem ChemPhys 18:27442-27449

61. Thom R (1972) Stabilité Structurelle et Morphogénèse Intereditions, Paris

62. Saunders VR, Dovesi R, Roetti C, Causà M, Harrison NM, Orlando R, Zicovitch-Wilson CM (1998) CRYSTAL98, User's manual Torino, Italy

63. Schäfer A, Huber C, Ahlrichs R (1994) Fully optimized contracted Gaussian basis sets of triple zeta valence quality for atoms Li to Kr J Chem Phys 100:5829-5835

64. Perdew JP, Wang Y (1992) Accurate and simple analytic representation of the electron-gas correlation energy Phys Rev B 45:13244-13249

65. Gatti C (1998) Topond98 manual CNR-CSRSRC, Milano, Italy

66. Noury S, Krokidis X, Fuster F, Silvi B (1999) Computational tools for the electron localization function topological analysis. Comput in Chem 23:597-604

67. (2002) Amira 3.0 TGS, Template Graphics Sofware, Inc., San Diego, USA

68. Gschneidner Jr KA (1964) Physical Properties and Interrelationships of Metallic and Semimetallic Elements in Seitz F, Turnbull D (eds.) Solid State Physics vol. $16275-$ 426 Academic Press

69. Hanfland M, Loa I, Syassen K, Schwarz U, Takemura K (1999) Equation of state of lithium to $21 \mathrm{GPa}$ Solid State Communications 112:123 - 127

70. Ormeci A, Rosner H, Wagner F, Kohout M, Grin Y (2006) Electron Localization Function in Full-Potential Representation for Crystalline Materials J Phys Chem A 110:1100-1105

71. Savin A, Becke AD, Flad J, Nesper R, Preuss H, von Schnering HG (1991) A New Look at Electron Localization Angew Chem Int Ed Engl 30:409

72. Savin A, Jepsen O, Flad J, Andersen OK, Preuss H, von Schnering HG (1992) Electron Localization in the Solid-State Structures of the Elements: the Diamond Structure Angew Chem Int Ed Engl 31:187-190

73. Savin A, Nesper R, Wengert S, Fässler TF (1997) ELF: The Electron Localization Function Angew Chem Int Ed Engl 36:1809-1832

74. Alikhani ME, Shaik S (2006) A topological study of the ferromagnetic "no-pair bonding" in maximum-spin lithium clusters: $\mathrm{Li}-\mathrm{n}+1(\mathrm{n})(\mathrm{n}=2-6)$ Theor Chem Acc 116:390-397

75. Contreras-García J, Martín Pendás A, Silvi B, Recio JM (2008) Useful applications of the electron localization function in high-pressure crystal chemistry J Phys Chem Solids 69:2204-2207

76. Contreras-Garcia J, Mori-Sanchez P, Silvi B, Recio JM (2009) A Quantum Chemical Interpretation of Compressibility in Solids J Chem Theory Comput 5:2108-2114 